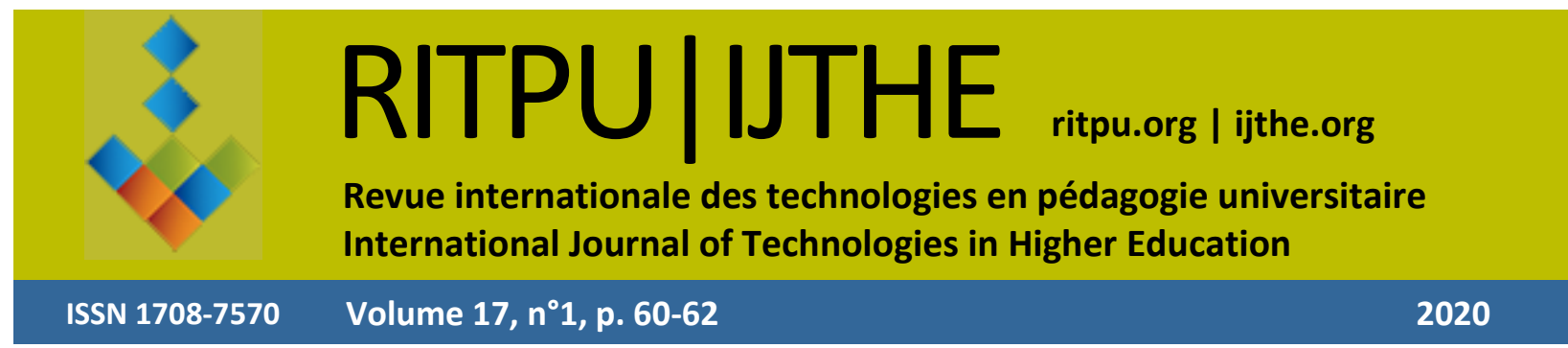

\title{
La culture du numérique, ou comment répondre aux besoins de l'enseignement et de l'apprentissage du réseau collégial?
}

Denis CHABOT

dchabot@ccdmd.qc.ca

Centre collégial de développement de matériel didactique (CCDMD)

\section{Digital Awareness or How to Answer the Needs of Teachers and Students of Quebec Colleges}

https://doi.org/10.18162/ritpu-2020-v17n1-12

Depuis sa fondation en 1993, le Centre collégial de développement de matériel didactique (CCDMD) a vu son catalogue s'enrichir constamment de ressources numériques destinées autant à l'enseignement qu'à l'apprentissage (REA). Ce mode de diffusion englobe l'ensemble de la production, les manuels imprimés étant également accessibles en format numérique, et il offre un potentiel pédagogique important. Au fil de l'évolution des connaissances en pédagogie, des besoins de la clientèle et des « révolutions » technologiques, le CCDMD maintient une tradition éditoriale basée sur la qualité de contenus validés, l'écoute des besoins et les meilleures pratiques. En considérant la culture du numérique dans son sens le plus large, nous tenterons de dégager les constantes dans le processus d'accompagnement des auteurs (figure 1) et de production des ressources (figure 2) en prenant en compte les besoins des utilisateurs et la contribution des différentes personnes-ressources appelées en renfort.

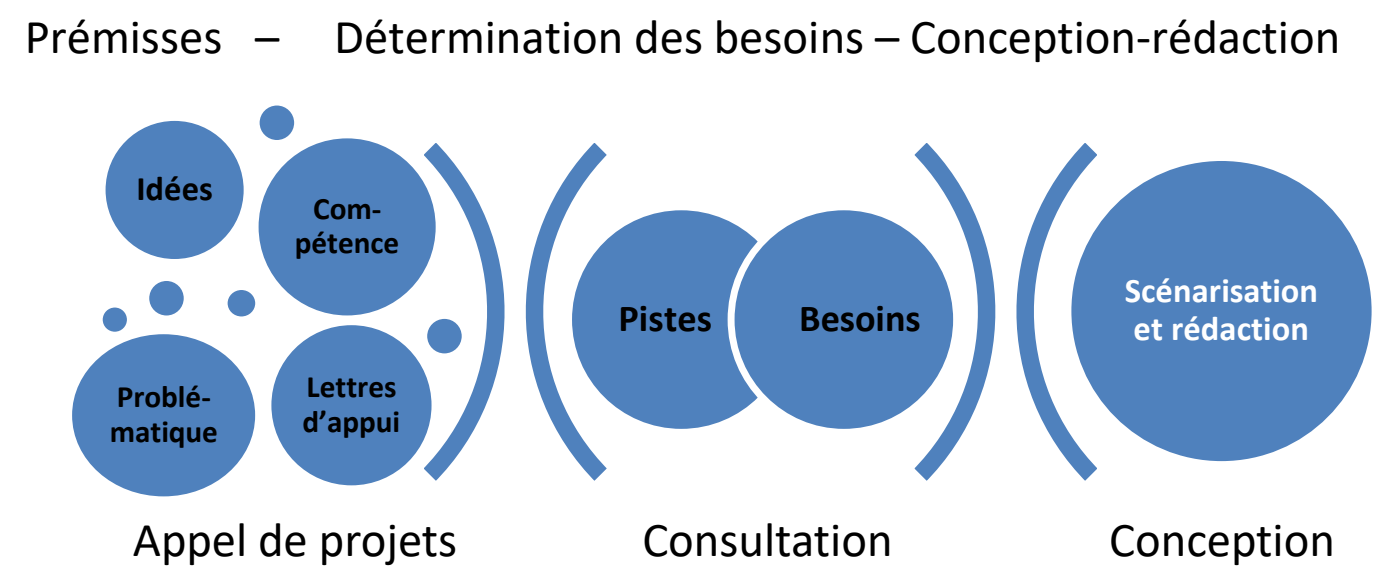

Figure 1

Processus d'accompagnement des auteurs 


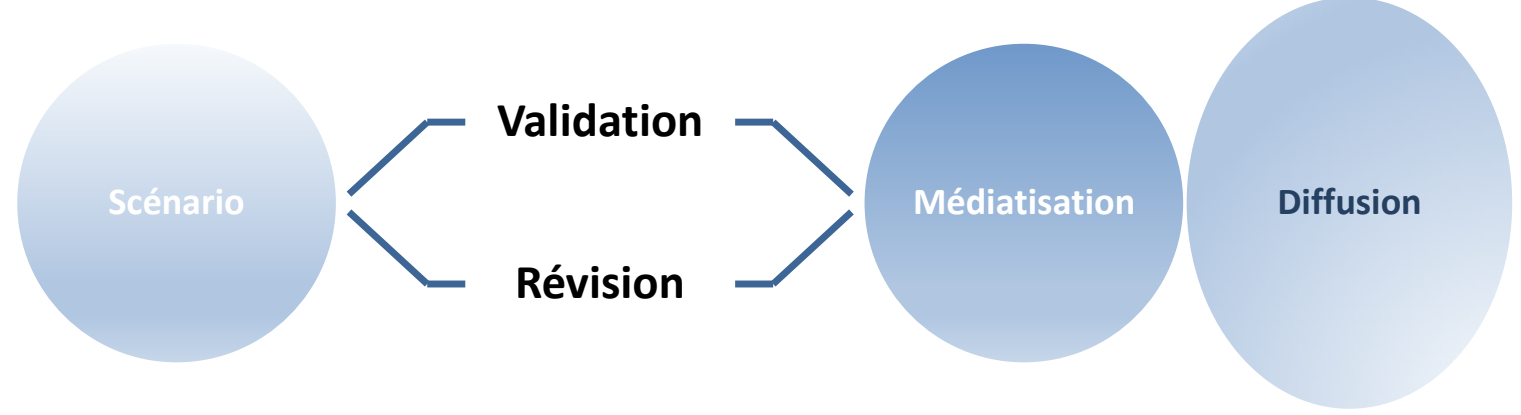

Figure 2

Production ou développement de la ressource

\section{Appel de projets}

Regardons d'abord le processus aux différentes étapes du projet. Si l'on réfère à la question posée par le titre du présent article, la première condition pour répondre à un besoin consiste à se donner les moyens de l'entendre. Le premier outil utilisé pour recevoir les questions ou les problèmes soulevés par les enseignants du réseau collégial québécois et les pistes de solution qu'ils proposent est l'appel de projets annuel. Le formulaire de l'appel de projets doit être rempli par un enseignant, que nous encourageons à consulter les ressources pédagogiques de son collège et les responsables du CCDMD. Les lettres d'appui rédigées par les différents établissements visés apporteront une première série de données faisant écho à l'initiative d'un seul département. Le projet sera ensuite évalué par un comité de sélection. Cette évaluation initiale à l'échelle du réseau collégial constitue une étape déterminante dans le processus itératif de validation. Les bonnes idées doivent alors reposer sur une problématique et des pistes de solution élaborées au regard de données probantes. Au moment d'accepter un projet, le comité de sélection peut suggérer des pistes alternatives ou des contingentements relativement à la faisabilité. Les meilleures idées partent d'un problème précis et doivent reposer sur une solution pertinente et réaliste. Le choix technologique ou médiatique demeure un moyen pour répondre au besoin; il ne doit pas devenir une fin en soi.

\section{Consultation}

Une fois retenu par le comité de sélection, le projet passera à l'étape de la consultation des utilisateurs potentiels : les enseignants de plusieurs établissements offrant le même programme. Que cette collecte de données prenne la forme d'un sondage ou d'un groupe de discussion, nous souhaitons la participation de quelques étudiants. La présentation d'un prototype, d'une table des matières, d'un chapitre ou d'un scénario permet de valider différents éléments relatifs à la forme autant qu'au contenu proposé. Ainsi, le format définitif peut varier par rapport à l'idée initiale. Une série de questions conçues pour des fichiers PDF destinés à l'impression peut migrer vers un questionnaire interactif réalisé à l'aide de Netquiz Web.

\section{Conception et rédaction}

L'essentiel de la contribution de l'enseignant qui se lance dans l'élaboration d'une REA se concentre à cette étape fondamentale de la création. Il faut choisir les mots, les images et les séquences d'apprentissage afin de les agencer à l'idée principale et aux objectifs du projet. En plus de l'effort de création et de rédaction, il faut planifier, dresser des listes et commencer à didactiser les éléments. L'accompagnement éditorial joue un rôle important à cette étape, qui 
précède la production ou la médiatisation. Sans que l'on ait toujours en main la maquette définitive, la mise en forme ou la présentation de l'information doit déjà faire partie de l'équation. Concevoir et rédiger en prévision d'un manuel de plusieurs centaines de pages, pour quelques pages Web ou pour des dialogues qui devront être joués par des comédiens repose sur des règles différentes. Depuis toujours au CCDMD, il s'est avéré utile de s'adjoindre les services de spécialistes pour les trois tâches suivantes : validation scientifique, validation pédagogique et révision linguistique. Mais compte tenu de la diversité des formes que prennent les REA, des « spécialistes » liés à la forme devraient aussi avoir à intervenir avant l'entrée en production pour différentes tâches: analyse informatique, test d'utilisabilité, scénarisation, architecture de l'information, ergonomie, mise en page, etc.

\section{Production}

Au moment de déléguer la réalisation de certaines étapes de la création, l'éditeur doit demeurer le plus près possible du travail de ces professionnels afin de bien les intégrer au projet. L'auteur devra alors valider le travail de ces nouveaux collaborateurs. Depuis quelques décennies déjà, la médiatisation des contenus numériques offre un grand nombre de possibilités. Devant l'éventail des possibles, la nature des besoins exprimés doit aider à conserver une certaine direction. Des choix affirmés en ce qui concerne le ton, le style et même la couleur aident à orienter les élans créatifs; les règles de l'art et les principes d'accessibilité font le reste. Que cela concerne la mise en page d'un court texte destiné à une page Web ou le tournage de scènes en vidéo 360 avec des comédiens qui interagissent, une présence active et un suivi soutenu s'avèrent essentiels pour conserver l'unité et l'intégrité de la ressource. Il est important de communiquer à tous les membres de l'équipe de production les objectifs généraux du projet et son contexte d'utilisation. La charte graphique d'une ressource destinée aux élèves du secondaire ne sera pas nécessairement la même que celle conçue pour les étudiants de l'enseignement supérieur.

Les conditions propices à la réalisation de ressources numériques de qualité reposent sur un écosystème éditorial riche et diversifié. Le patrimoine éducatif, le savoir-faire et les pratiques innovantes issus de cet écosystème participent au Plan d'action numérique en éducation et en enseignement supérieur (PAN) : notamment au niveau de la mesure 11 de l'orientation 2 qui vise à soutenir l'acquisition et le dévelopement de ressources éducatives numériques (REN). Les outils issus de la culture du numérique permettent de faire plus de choses et de les faire de manière différente, mais il ne suffit pas de colliger beaucoup de données, de l'information fiable et des modèles scientifiques afin de partager ces recueils dans la première application infonuagique proposée par l'économie du partage. La richesse d'une ressource numérique ne devrait-elle pas se mesurer à la qualité des apprentissages qu'elle permet d'envisager? En terminant, nous croyons essentiel de souligner la place de plus en plus prépondérante que la collaboration prend dans un tel écosystème. Les ressources éducatives libres (REL) nous incitent à rêver d'un patrimoine éducatif de grande qualité à la hauteur du potentiel offert par la culture du numérique. 